\section{Radionuclide neutron source trajectories in the closed nuclear fuel cycle*}

\begin{abstract}
The highest efficiency in the usage of nuclear energy resources can be implemented in fast breeder reactors of generation IV. It is achieved thanks to the ability of consuming minor actinides (MAs) in energy production. One of the options to use this benefit is full recycling of MAs to close the nuclear fuel cycle. Monte Carlo burn up (MCB), an integrated burn-up calculation code, deals with the complexity of the burn-up process which is applied to the European Lead-cooled Fast Reactor (ELFR). MCB uses continuous energy representation of cross section and spatial effects of full core reactor model; however, it automatically calculates nuclide production in all possible reactions or decay channels. Multi-recycling of MAs can cause an intensified build-up of curium, berkelium and californium. Some of their isotopes are strong neutron emitters from spontaneous fission, which hinders fuel recycling. The implementation of a novel methodology for trajectory period folding allows us to trace the life cycle of crucial MAs from the beginning of the reactor life towards the state of adiabatic equilibrium. The result of the analysis performed is presented, showing the sources of strong contribution to the neutron production rate. The parametric sensitivity analysis method for selected nuclide reactions is applied, revealing sensitivity of transmutation chains for the production of neutron emitter isotopes.
\end{abstract}

Keywords: MCB • transmutation $\bullet$ equilibrium fuel cycle $\bullet$ LFR $\bullet$ trajectory period folding

P. Stanisz ${ }^{\bowtie}$, J. Cetnar, M. Oettingen

Department of Nuclear Energy

Faculty of Energy and Fuels

AGH University of Science and Technology

30 A. Mickiewicza Ave., 30-059 Krakow, Poland

E-mail: pstanisz@agh.edu.pl

Received: 20 November 2017

Accepted: 10 December 2018

\section{Introduction}

The highest efficiency in the usage of nuclear energy resources can be implemented in fast breeder reactors of generation IV. It is achieved thanks to the ability of consuming minor actinides (MAs) in energy production. One of the options to use this benefit is MA reprocessing to close the nuclear fuel cycle. It is a state in which the reactor is supplied only by natural or depleted uranium from the outside. The core consumes self-produced fissile fuel and produces only fission products. In the sense that there is no exchange of transuranics (TRUs) with the environment during the whole operation, this concept corresponds to the "adiabatic fuel cycle" [1]. By using this method, it is possible to reach the equilibrium state of the reactor core. The numerical experiment was applied to the European Lead-cooled Fast Reactor (ELFR) - a design for an industrially sized reactor prepared at the National Agency for New Technologies, Energy and the Environment (ENEA). The assessment of the adiabatic equilibrium procedure was performed using Monte

\footnotetext{
* This paper is based on a poster presentation showed at NUTECH-2017 Conference, 10-13 September 2017, Kraków Poland.
} 
Carlo burn up (MCB) [2], an integrated burn-up calculation code which deals with the complexity of the burn-up process. Calculations include continuous energy representation of cross section, spatial effects of full core reactor model and nuclide production in all possible reactions or decay channels. So far, the outcomes of the numerical modelling of the ELFR design, such as adiabatic composition, criticality, breeding gain, power and burn-up, have been presented in various papers $[3,4]$ and reports $[5,6]$.

In this paper, we focus on the effect of the long exposure time for neutron radiation through MA multi-recycling, which can cause an intensified build-up of curium $(\mathrm{Cm})$, berkelium $(\mathrm{Bk})$ and californium (Cf). Some of their isotopes are strong neutron emitters from spontaneous fission, which can hinder fuel recycling. The implementation of a novel methodology for trajectory period folding allows us to trace the life cycle of crucial MAs from the beginning of the reactor life towards the state of adiabatic equilibrium.

\section{Trajectory period-folding method}

The depletion problem describes the evolution of nuclide composition over time. One of the numerical solutions of this problem is the linear chain approach [7], which represents series of physically occurring nuclide transitions in physical meaning. Transition chains preserve all quantitative information about the transmutation process. So far, the linear chain approach has been applied to a single computational step, after which a new nuclide vector is created and the information about the transmutation process is lost after more than one step. The novel approach presented here allows for tracking of nuclear trajectories during the entire fuel evolution. Trajectories prepared for each computing time step are combined in the process of time period folding, which allows to identify a physical pathway for the build-up of isotopes during the entire fuel evolution. The process of composed joint trajectory distribution is recursively repeated for each last nuclide in the previously defined trajectory. The procedure that corresponds to the trajectory period folding is explained here using an example.

The procedure works as follows; an example trajectory is described by the transmutation chain:

$$
{ }^{238} \mathrm{U} \rightarrow{ }^{239} \mathrm{U} \rightarrow{ }^{239} \mathrm{~Np} \rightarrow{ }^{239} \mathrm{Pu}
$$

The procedure of building trajectories starts with finding all possible transmutations which can occur in the first and the second computed period. The first outermost iteration of the procedure selects the ancestor nuclide from the first period, if the initial fuel vector contains more than one nuclide. In the example, we consider only one initial ancestor nuclide, which starts with ${ }^{238} \mathrm{U}$. The second-level iteration selects the trajectory which is described by a linear transmutation chain containing the same chained series as the period-folded transmutation chain. Let us suppose that the selected $k^{\text {th }}$ trajectory is described by the following transmutation chain:

$$
{ }^{1} T_{1, l(k)}^{(k)}\left(t_{1}\right):{ }^{238} \mathrm{U} \rightarrow{ }^{239} \mathrm{U} \rightarrow{ }^{239} \mathrm{~Np}
$$

where the superscript on the left indicates period number, the superscript on the right indicates the trajectory number (the $k^{\text {th }}$ trajectory), the first subscript of the trajectory represents the first family, which starts with the ${ }^{238} \mathrm{U}$ ancestor, and the second subscript is the descendant nuclide $\left({ }^{239} \mathrm{~Np}\right)$ in the $k^{\text {th }}$ trajectory.

The reaction at the end of the first period ends with ${ }^{239} \mathrm{~Np}$; therefore, period-folded trajectory should be prolonged by the trajectory from the second period starting with ${ }^{239} \mathrm{~Np}$. The third-level iteration is performed by searching for the family from the second period, which starts with ${ }^{239} \mathrm{~Np}$. In the example, index 3 is assigned to ${ }^{239} \mathrm{~Np}$ family. From the set of trajectories (family) starting with ${ }^{239} \mathrm{~Np}$, the fourth-level iteration is performed over a set of trajectories by looking for the remaining matching chain series. In our example, the desired trajectory is the $h^{\text {th }}$ trajectory, which corresponds to the transmutation trajectory for neptunium over the second period.

$$
{ }^{2} T_{3, l(h)}^{(h)}\left(t_{2}\right):{ }^{239} \mathrm{~Np} \rightarrow{ }^{239} \mathrm{Pu}
$$

So far, one possible path has been found in which ${ }^{238} \mathrm{U}$ over two periods transmutes to ${ }^{239} \mathrm{Pu}$ over transmutation chain (4), where ${ }^{1} T^{(k)}{ }_{1, l(k)}\left(t_{1}\right)$ amount of ${ }^{238} \mathrm{U}$ transmutes through $k^{\text {th }}$ trajectory to ${ }^{239} \mathrm{~Np}$ over the first period, while ${ }^{2} T^{(h), l(h)}\left(t_{2}\right)$ amount of ${ }^{239} \mathrm{~Np}$ transmutes over the second period to ${ }^{239} \mathrm{Pu}$. Thus, after two periods, the joint transition is equal to $\left.{ }^{1} T^{(k) l, l(k)}\left(t_{1}\right) \cdot{ }^{2} T^{(h)}\right)_{3, l(h)}\left(t_{2}\right)$.

$$
\begin{array}{r}
{ }^{1,2} T_{3, l(g)}^{(g)}\left(t_{1}+t_{2}\right)={ }^{1} T_{1, l(k)}^{(k)}\left(t_{1}\right) \cdot{ }^{2} T_{3, l(h)}^{(h)}\left(t_{2}\right): \\
{ }^{238} \mathrm{U} \rightarrow{ }^{239} \mathrm{U} \rightarrow{ }^{239} \mathrm{~Np} \rightarrow{ }^{239} \mathrm{Pu}
\end{array}
$$

The presented path is one of the four combinations that give the same period-folded trajectory (5); thus, the total sum of all combinations between the transitions must be considered:

(5)

$$
\begin{aligned}
& 1^{\text {st }} \text { period } \\
& { }^{238} \mathrm{U} \rightarrow{ }^{239} \mathrm{U} \rightarrow{ }^{239} \mathrm{~Np} \rightarrow{ }^{239} \mathrm{Pu}
\end{aligned}
$$

The procedure is repeated until all possible combinations are found. The sum of all possible joint transitions describes the period-folded trajectory transition (or shortly: folded trajectories).

\section{Reference core and fuel cycle strategy implementation}

The core proposed as the application of the equilibrium approach is designed to have $1500 \mathrm{MW}$ (600 MWe) [8]. The claim for the ELFR in the adiabatic equilibrium state has imposed a novel procedure for reactor designing, which is known as the new paradigm for nuclear power [1]. In this procedure, the initial geometry is designed firstly from the 

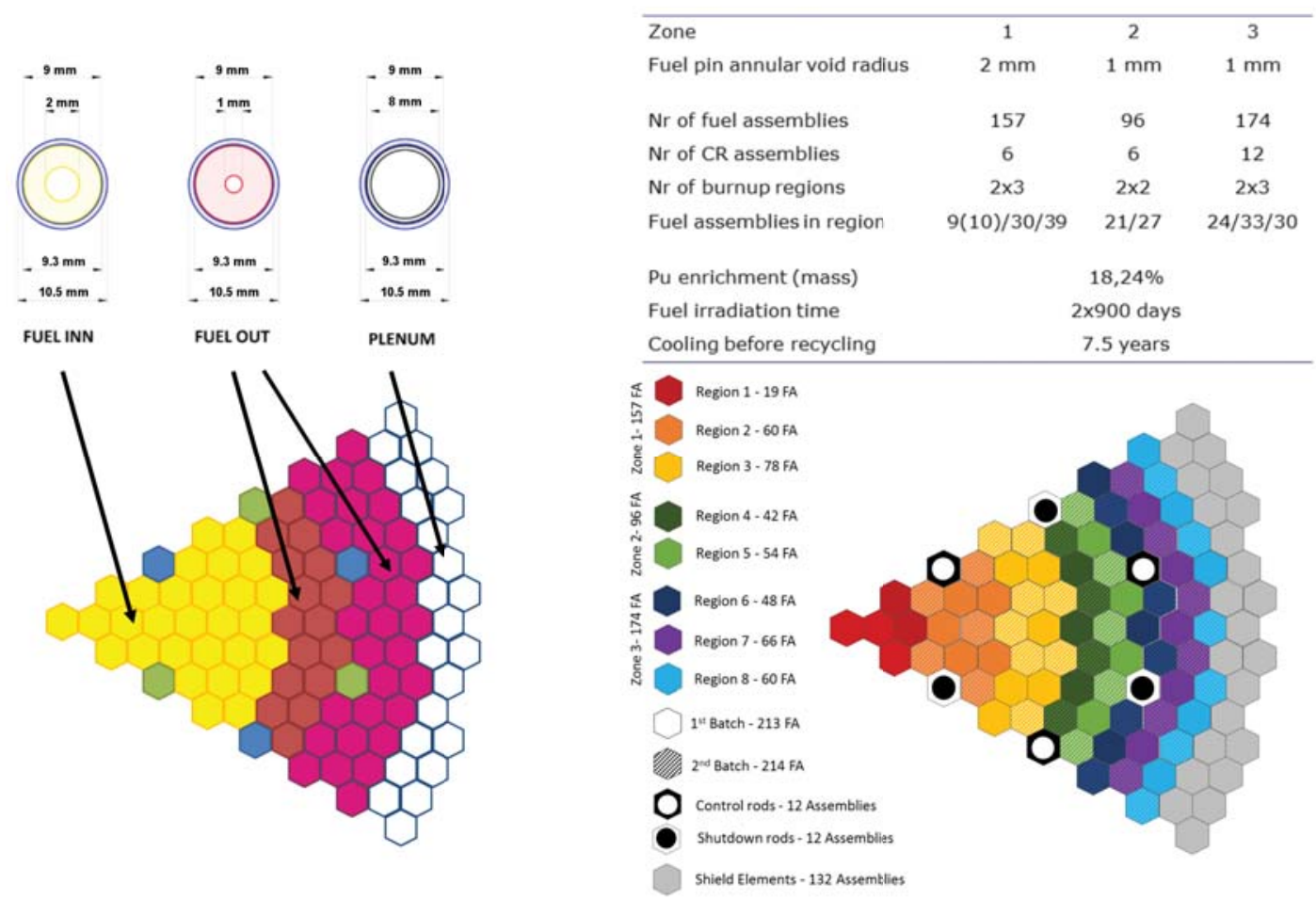

Fig. 1. ELFR core properties and configuration with a division of the pin annular void and burn-up zones. Dashed and coloured elements represent adequate burn-up zones together with a distinction between the first and the second batch.

initial geometry of the elementary cell, considering thermal-hydraulic parameters. The derived geometry defines the neutron spectrum, which affects the equilibrium composition of the nuclear fuel which is determined. Finally, the number of elementary cells can be adjusted by manipulating the fuel volume fraction in the elementary cell along with the fuel composition to find the criticality, power and size of the core [3]. Figure 1 (scheme on the left) shows an alternative concept of fissile material distribution in the core. Instead of providing different enrichment zoning, ENEA and Joint Research Centre (JRC) proposed one single fuel enrichment during both irradiation and processing, with optimization of zoning and annual dimension referred here as the pin annular void zoning. Figure 1 (scheme on the right) shows the division of burn-up zones used in the calculation by the MCB code. Each region is additionally divided into 10 axial zones, creating 160 burn-up zones in total. The ELFR core parameters were presented in detail in Ref. [3].

In the closed cycle, fuel composition changes over time, approaching the equilibrium. Fuel residence time in the reactor has an overwhelming influence on the production of higher actinide nuclides. Traditional validation exercises compare measured parameters against experiments and then set envelopes of operation, so that problems cannot arise [9]. In a system with long campaign, uncertainties of computer predictions, resulting from nuclear cross section, propagate directly to the determined nuclide production. In turn, important nuclear safety parameters, such as criticality, heat production and radiation level, depend on the spent fuel inventory. Precise identification of governing transmutation chains together with their impact on the safety-related nuclide production is the first step towards the better understanding and estimation of respective effects in the fast nuclear system under the multistep approximation. The build-up of higher actinide nuclides, induced by fast neutron irradiation, can be reached in several transmutation chain trajectories. Thanks to the trajectory period method, with quantitative determination, we answer to the question of how and which transmutation chains are involved in the safety-related nuclide production.

The results obtained here based on one single batch evolution (in this case - the second one) are shown in Fig. 2 applied into the ELFR design [10]. The evolution of nuclide density is calculated with the extension of the trajectory set prepared for every zone and time step (development of the procedure is described in Ref. [3]). Therefore, trajectories and nuclide vectors are automatically adjusted to the evolving transmutation conditions. Trajectories are followed for every zone. The period-folding procedure is performed for each subsequent computational step. Trajectories belonging to the investigated batch are mixed after each fuel unload period; thus, folded trajectories are averaged before recycling. After the cooling period, fuel is loaded into the reactor core and folded trajectories are again followed separately for each burn-up zone with consecutive steps. The results presented show folded transmutation chains of one single batch at the end of each cooling period, before the recycling and preparation of new initial fuel.

The simulation performed by MCB used JEFF-3.1 nuclear data library [11]. One hundred and fifty active cycles and 50000 neutron histories per cycle were used to gather the reaction rate in burnable zones. The libraries incorporate the decay scheme for 


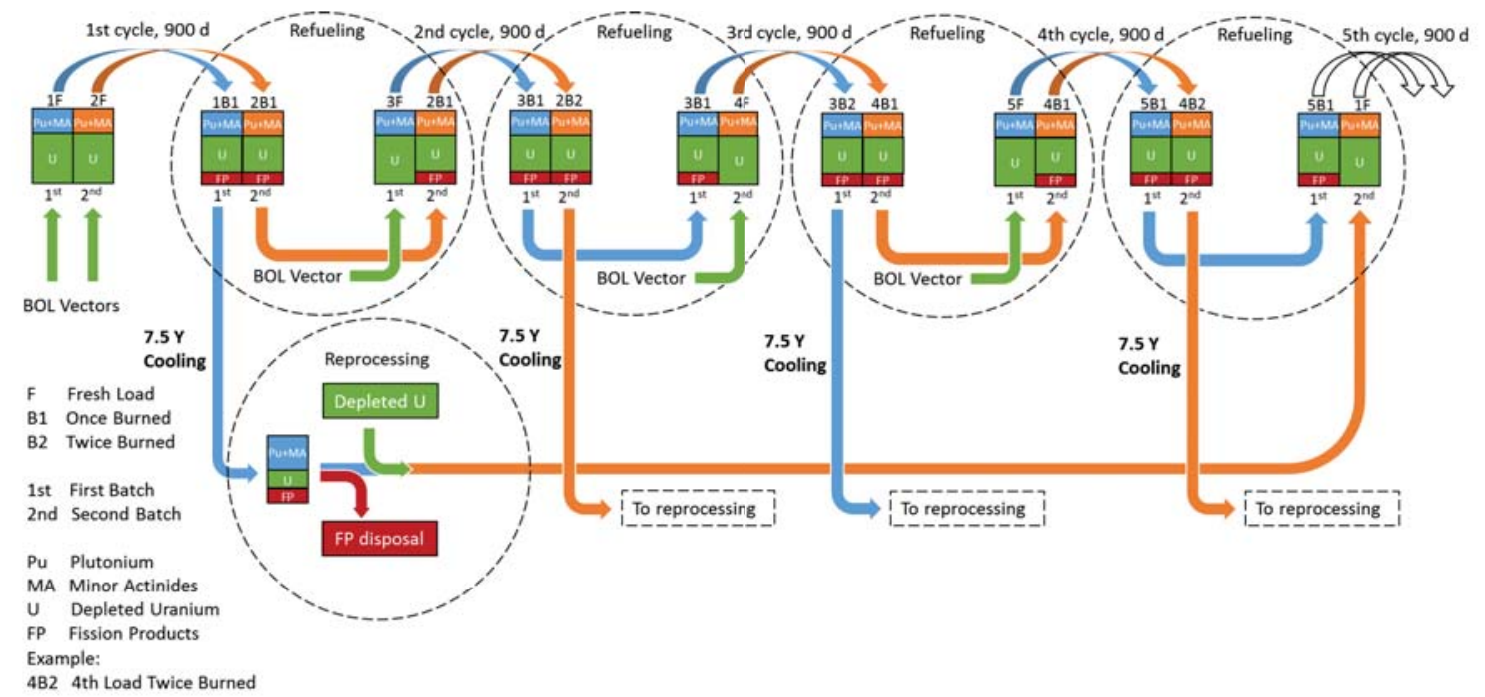

Fig. 2. Flowchart of MCB adiabatic procedure used to reach the equilibrium state.

about 2400 isotopes based on the table of isotopes [12]. Initially, to establish the equilibrium state, we investigated the changes from cycle to cycle in main isotopes [3], which primarily influenced the main parameter of the core characteristics, which can be found constant after approximately 50 years. In the performed scenario, the initial value of MA concentration was assumed at $1.347 \%$, which corresponds to the equilibrium composition calculated in the previous analysis [3]. The MA composition was typical for the light-water reactor (LWR) spent fuel. The purpose of this choice was to obtain the reference fuel composition with fixed MA fraction. At the end of each cooling cycle, depleted uranium replaces removed fission products in equal mass. The cycle analysis results in the transmutation of non-fission reaction coupled with subsequent decay, which leads to a vector that converges to the equilibrium vector. Fuel multi-recycling in adiabatic cycles can cause some hazards during fuel reprocessing. Self-breeding cores have an impact on the production of higher actinides. Higher actinides grow from zero to their equilibrium value at their adequate time rate. The comparison between the fuel compositions from fresh load and back-end fuel (adopted adiabatic state after 124.2 years) is summarized in Table 1. By performing standard calculations, we cannot observe detailed mass flows between the elements. A description of transmutation performed in a standard analysis without period trajectories can be based on qualitative, but not quantitative results. The application of the trajectory period-folding method allows to quantitatively assess physical transmutation chains which participate in the production of safety-related nuclides. Two nuclides were chosen for the investigation of build-up pathways: ${ }^{244} \mathrm{Cm}$ and ${ }^{252} \mathrm{Cf}$. Both nuclides have a branching reaction resulting in spontaneous fission.

Recognition of the production of safety-related nuclides is important for the estimation of their concentration together with their uncertainty and the indication of the crucial cross section in determining the equilibrium state. In particular, higher MAs have to be estimated with greater confidence.
Resultant decay heat and radiation terms from higher MAs produced through the newly proposed fuel cycles will have to meet future regulatory frameworks. The newly proposed cycles may require new chemical processing plants, different from those currently in use, which will have to meet new safety requirements. Higher decay heat requires either more intensive fuel cooling or less fuel transported, stored or processed at a time, compared with the existing facilities. This will lead to greater costs [13]. Similarly, different radiation source terms,

Table 1. Initial and final batch's fuel composition

\begin{tabular}{|c|c|c|}
\hline Isotope & $\mathrm{BOL}[\mathrm{g}]$ & EOL $[\mathrm{g}]$ \\
\hline$\overline{\mathrm{U}}$ & $2.14 \cdot 10^{7}$ & $2.00 \cdot 10^{7} / 2.15 \cdot 10^{7 *}$ \\
\hline${ }^{234} \mathrm{U}$ & $6.42 \cdot 10^{2}$ & $5.71 \cdot 10^{4}$ \\
\hline${ }^{235} \mathrm{U}$ & $8.64 \cdot 10^{4}$ & $2.23 \cdot 10^{4}$ \\
\hline${ }^{236} \mathrm{U}$ & $2.14 \cdot 10^{3}$ & $3.57 \cdot 10^{4}$ \\
\hline${ }^{238} \mathrm{U}$ & $2.13 \cdot 10^{7}$ & $1.99 \cdot 10^{7} / 2.14 \cdot 10^{7 *}$ \\
\hline${ }^{237} \mathrm{~Np}$ & $1.36 \cdot 10^{4}$ & $2.65 \cdot 10^{4}$ \\
\hline $\mathrm{Pu}$ & $4.82 \cdot 10^{6}$ & $4.80 \cdot 10^{6}$ \\
\hline${ }^{238} \mathrm{Pu}$ & $1.13 \cdot 10^{5}$ & $1.16 \cdot 10^{5}$ \\
\hline${ }^{239} \mathrm{Pu}$ & $2.74 \cdot 10^{6}$ & $2.67 \cdot 10^{6}$ \\
\hline${ }^{240} \mathrm{Pu}$ & $1.30 \cdot 10^{6}$ & $1.69 \cdot 10^{6}$ \\
\hline${ }^{241} \mathrm{Pu}$ & $2.94 \cdot 10^{5}$ & $1.18 \cdot 10^{5}$ \\
\hline${ }^{242} \mathrm{Pu}$ & $3.71 \cdot 10^{5}$ & $2.07 \cdot 10^{5}$ \\
\hline${ }^{244} \mathrm{Pu}$ & 0 & $3.19 \cdot 10^{0}$ \\
\hline Am & $3.29 \cdot 10^{5}$ & $2.93 \cdot 10^{5}$ \\
\hline${ }^{241} \mathrm{Am}$ & $2.70 \cdot 10^{5}$ & $2.08 \cdot 10^{5}$ \\
\hline${ }^{242 \mathrm{~m}} \mathrm{Am}$ & $9.11 \cdot 10^{2}$ & $1.55 \cdot 10^{4}$ \\
\hline${ }^{243} \mathrm{Am}$ & $5.79 \cdot 10^{4}$ & $6.96 \cdot 10^{4}$ \\
\hline $\mathrm{Cm}$ & $1.53 \cdot 10^{4}$ & $4.87 \cdot 10^{4}$ \\
\hline${ }^{242} \mathrm{Cm}$ & 0 & $3.77 \cdot 10^{1}$ \\
\hline${ }^{243} \mathrm{Cm}$ & $2.39 \cdot 10^{2}$ & $5.35 \cdot 10^{2}$ \\
\hline${ }^{244} \mathrm{Cm}$ & $1.09 \cdot 10^{4}$ & $3.06 \cdot 10^{4}$ \\
\hline${ }^{245} \mathrm{Cm}$ & $4.14 \cdot 10^{3}$ & $1.06 \cdot 10^{4}$ \\
\hline${ }^{246} \mathrm{Cm}$ & $3.23 \cdot 10^{2}$ & $5.63 \cdot 10^{3}$ \\
\hline${ }^{247} \mathrm{Cm}$ & $6.05 \cdot 10^{0}$ & $9.73 \cdot 10^{2}$ \\
\hline${ }^{248} \mathrm{Cm}$ & $4.93 \cdot 10^{-1}$ & $3.49 \cdot 10^{2}$ \\
\hline $\mathrm{HM}$ & $2.66 \cdot 10^{7}$ & $2.52 \cdot 10^{7} / 2.67 \cdot 10^{7 *}$ \\
\hline
\end{tabular}

BOL: beginning of life. EOL: end of life (after 124.2 years). HM: heavy metal. *Before and after admixing of depleted uranium. 


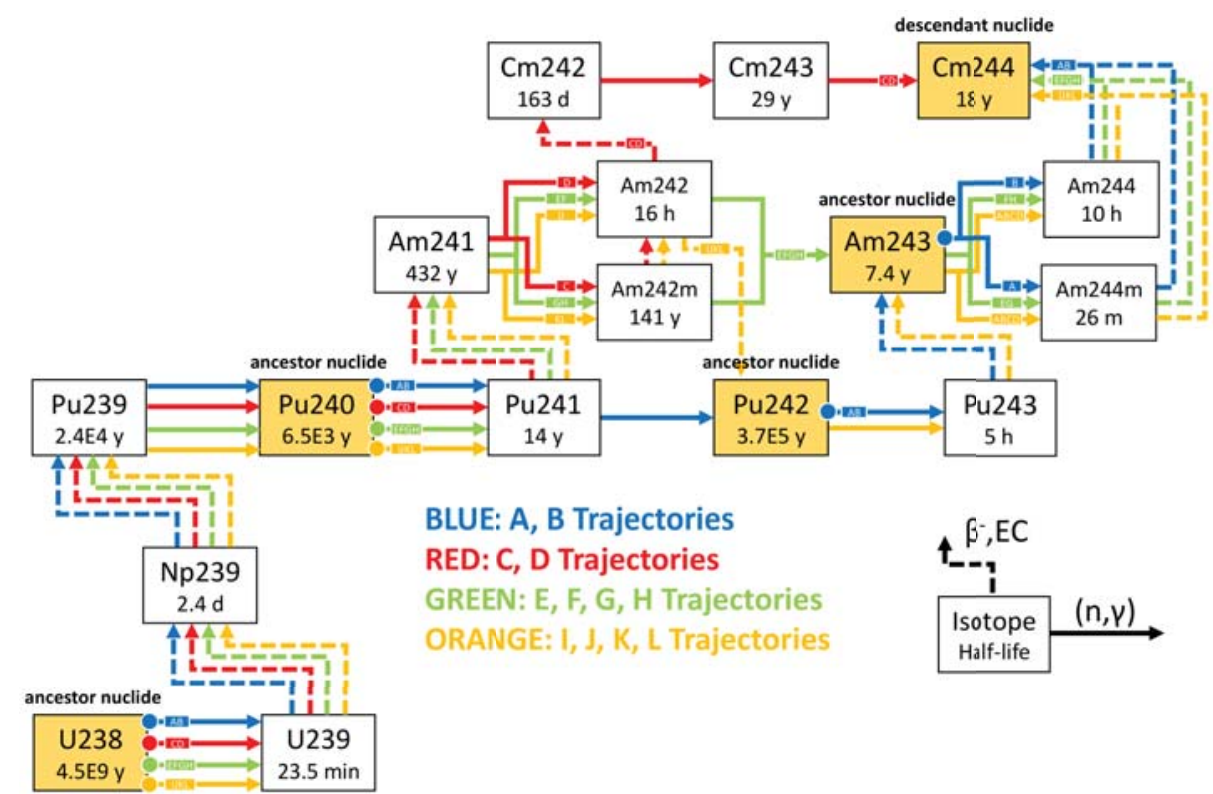

Fig. 3. Main trajectories producing ${ }^{244} \mathrm{Cm}$.

from gamma rays and/or neutrons, will lead to the increased expenses on the protection of workers in separation and fabrication facilities. In the future, facilities will have to be prepared for the expected fuel quantity and composition, taking into account their uncertainty. That is why minimizing the uncertainties affecting fuel composition is so important.

\section{Production of $\mathrm{Cm}-244$}

Curium-244 is the last gateway isotope to higher-mass MA [14] and one of the main sources of neutrons from spontaneous fission. For these reasons, it is important to recognize in detail the trajectories that lead to the production of ${ }^{244} \mathrm{Cm}$. Its main 12 transmutation chains are shown in Fig. 3. The mass evolution from the particular trajectories is presented in Fig. 4. The colours distinguish four main paths that the transmutations pass from nuclides ${ }^{238} \mathrm{U},{ }^{240} \mathrm{Pu},{ }^{242} \mathrm{Pu},{ }^{243} \mathrm{Am}$, which are presented at the beginning of life (BOL) to ${ }^{244} \mathrm{Cm}$. Two or four variants for each colour are caused by the reaction

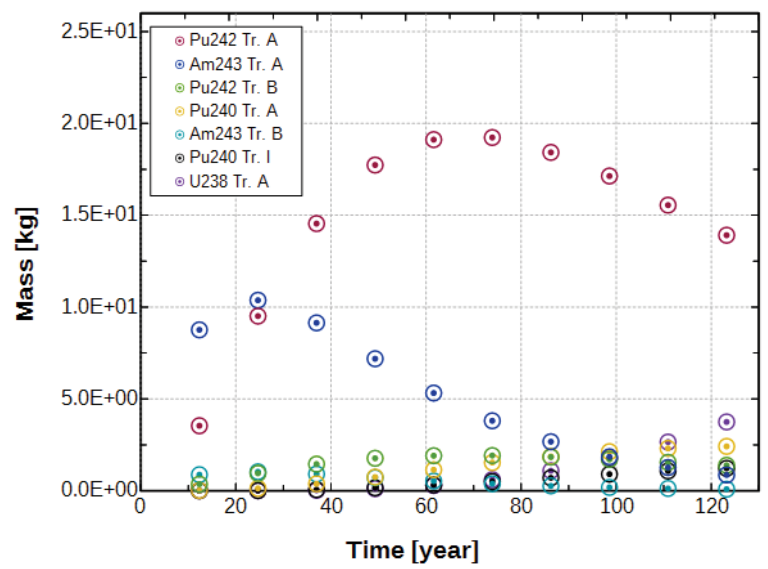

Fig. 4. Mass evolution produced from the most prominent trajectories leading to ${ }^{244} \mathrm{Cm}$. branching ratio of the production of ${ }^{242} \mathrm{Am} /{ }^{242 \mathrm{~m}} \mathrm{Am}$ and ${ }^{244} \mathrm{Am} /{ }^{244 \mathrm{~m}} \mathrm{Am}$.

The observed trajectories participate in the production of ${ }^{244} \mathrm{Cm}$ with different contributions, depending on their respective reactions that build the trajectories. The results are consistent with the supposition that for most of the time considered (124 years) ${ }^{244} \mathrm{Cm}$ concentration is produced mainly from ${ }^{242} \mathrm{Pu}$ and ${ }^{243} \mathrm{Am}$, rather than from ${ }^{240} \mathrm{Pu}$ and ${ }^{238} \mathrm{U}$ (which are supposed to increase their importance during following fuel cycles). The transmutation chains from ${ }^{242} \mathrm{Pu}$ and ${ }^{243} \mathrm{Am}$ are shorter, and there are fewer fission reactions and less decays on the way to ${ }^{244} \mathrm{Cm}$, compared with the trajectories starting from ${ }^{240} \mathrm{Pu}$ and ${ }^{238} \mathrm{U}$. The production from the first two nuclides is strongly dependent on their initial concentration coming from pressurized-water reactor's (PWR) MA load. The largest share in the production of ${ }^{244} \mathrm{Cm}$ comes from the ${ }^{242} \mathrm{Pu}$ trajectory A. It is responsible for the observable hump. To optimize the assessment of its maximal value, the reaction belonging to the responsible reactions, such as ${ }^{242} \mathrm{Pu} \stackrel{n_{\vartheta} \longrightarrow}{\longrightarrow}{ }^{243} \mathrm{Pu}$ and ${ }^{243} \mathrm{Am} \stackrel{n_{\vartheta} \rightarrow}{\longrightarrow}{ }^{244 \mathrm{~m}} \mathrm{Am}$, should be investigated.

\section{Production of Cf-252}

Californium-252, alongside ${ }^{244} \mathrm{Cm}$, is the main source of neutrons from spontaneous fission. ${ }^{252} \mathrm{Cf}$ ends with spontaneous fission for $3.09 \%$ branching of decays with 2.65-year half-life. The production of ${ }^{252} \mathrm{Cf}$ is connected mainly with successive neutron absorptions. This way of approaching ${ }^{252} \mathrm{Cf}$ is illustrated in Fig. 5. Corresponding mass evolution from the trajectories is presented in Fig. 6. Nuclides such as ${ }^{252} \mathrm{Cf}$, which are built through many successive neutron absorptions, are potentially burdened with higher uncertainty coming from neutron cross sections. This process is additionally hindered by higher uncertainties for trans-plutonium elements than for 


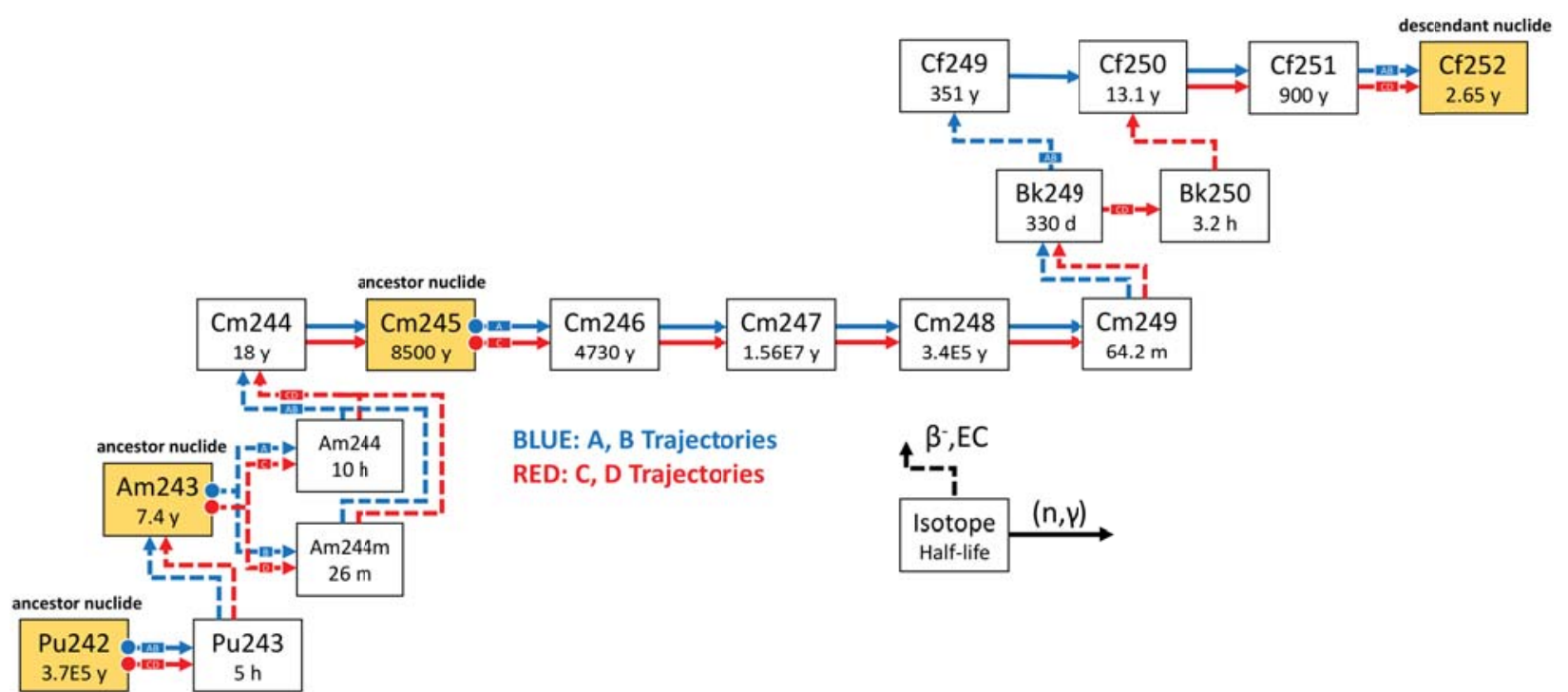

Fig. 5. Main trajectories producing ${ }^{252} \mathrm{Cf}$.

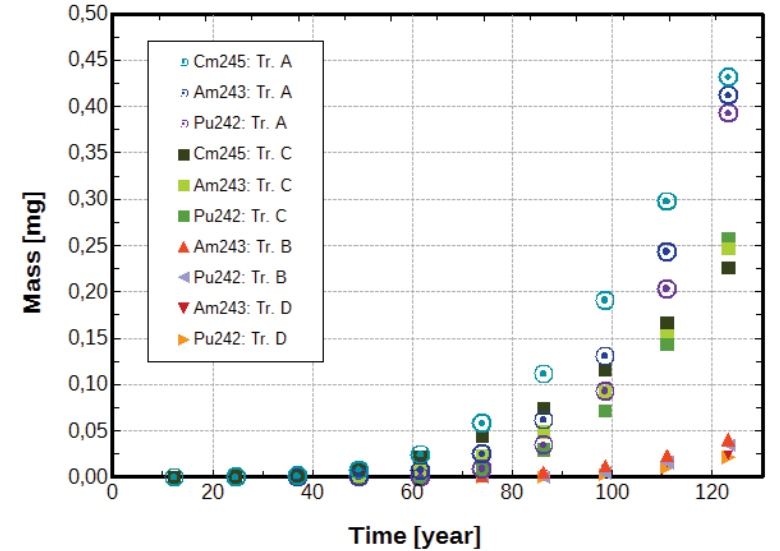

Fig. 6. Mass of ${ }^{252} \mathrm{Cf}$ undergoes spontaneous fission produced by the most prominent trajectories.

the lighter trans-uranium nuclides. These effects result in difficulties associated with measurements of cross sections.

The results present 10 trajectories starting from ${ }^{242} \mathrm{Pu},{ }^{243} \mathrm{Am}$ and ${ }^{245} \mathrm{Am}$. Nuclides such as ${ }^{240} \mathrm{Pu}$ and ${ }^{238} \mathrm{U}$ require much more time to reach their maximum in the production of ${ }^{252} \mathrm{Cf}$ (and its spontaneous fission fraction). This makes us think how much time is required to reach the equilibrium in which all products come from ${ }^{238} \mathrm{U}$. Trajectories are guided mainly by the reaction of absorption, which competes with the removal fission reaction and the decay of curium and californium. Non-fission reactions lead to successive heavier nuclides and finally to ${ }^{252} \mathrm{Cf}$. Only ${ }^{249} \mathrm{Bk}$ results in two possible transmutation channels which can change the production of ${ }^{252} \mathrm{Cf}$. From the analysis of a single reaction of ${ }^{249} \mathrm{Bk}$ and its effective transmutation branching, beta reaction occurs with $\sim 91 \%$ events and neutron capture with $\sim 9 \%$ events. This analysis does not consider consecutive reactions. Taking into account the trajectories that go through all reactions, we finally have $35-45 \%$ contribution that transmutes through ${ }^{249} \mathrm{Bk}$ neutron absorption, avoiding its beta decay. This is possible thanks to ${ }^{249} \mathrm{Cf}$ fission reaction, which reduces the production of ${ }^{252} \mathrm{Cf}$. Since ${ }^{249} \mathrm{Bk}$ decay half-time is well known, improvement in the cross section of both reactions on ${ }^{249} \mathrm{Bk}$ and ${ }^{249} \mathrm{Cf}$ will have to be performed to provide more information about the mass flow.

\section{Summary}

Improvement in the accuracy of capture and fission cross sections at intermediate and high neutron energies is required [15] for sufficiently accurate predictions of higher MA inventories, from ${ }^{241} \mathrm{Am}$ until ${ }^{252} \mathrm{Cf}$, which influence their final inventory. The application of the trajectory period-folding method allows for quantitative assessment of physical transmutation chains which participate in the production of gateway isotopes. A few conclusions can be drawn from the analysis performed:

- Period-folded trajectories quantitatively characterize how nuclides transmute over the computational period. Identification of the key trajectories in the formation of TRU elements is crucial for the understanding of the formation mechanism of notably radioactive isotopes.

- Improvement in nuclear cross sections should focus on those nuclides that are present in the trajectories and that participate in the production of MA inventory and safety-related nuclides.

- Apart from the assessment of transmutation reactions leading to higher actinide nuclides by neutron capture, other reactions, such as fission, should also be assessed. Fission reactions, like other removal reactions from the considered chain, compete with capture. Therefore, they should also be analysed quantitatively, given their impact on the finally spent fuel composition.

- The parametric sensitivity analysis should be the next step towards the verification of nuclear data. Interesting reactions can be checked from the point of view of their influence on the transmutation dynamics. This analysis should finally indicate the reaction with the highest influence.

- The production of ${ }^{244} \mathrm{Cm}$ is strongly dependent on the initial concentration of ${ }^{243} \mathrm{Am}$ and ${ }^{242} \mathrm{Pu}$. 
Both nuclides (through their A trajectories) are responsible for the growth of ${ }^{244} \mathrm{Cm}$ over the first 80 years.

- ${ }^{252} \mathrm{Cf}$ is formed through main transmutation pathways during the multicycle reloading scheme starting from the identified isotopes of ${ }^{242} \mathrm{Pu}$, ${ }^{243} \mathrm{Am}$ and ${ }^{245} \mathrm{Cm}$.

Acknowledgments. The research was partially supported by PL Grid Infrastructure available at the Academic Computer Centre CYFRONET AGH. In addition, partial financial support of this study under the grant agreement 11.11.210.377 by the Polish Ministry of Science and Higher Education is kindly acknowledged.

\section{References}

1. Artioli, C., Grasso, G., \& Petrovich, C. (2010). A new paradigm for core design aimed at the sustainability of nuclear energy: the solution of the extended equilibrium state. Ann. Nucl. Energy, 37, 915-922. https:// doi.org/10.1016/j.anucene.2010.03.016.

2. Oettingen, M., Cetnar, J., \& Mirowski, T. (2015). The MCB code for numerical modeling of fourth generation nuclear reactors. Comput. Sci., 16(4), 329-350.

3. Stanisz, P., Oettingen, M., \& Cetnar, J. (2016). Monte Carlo modeling of Lead-Cooled Fast Reactor in adiabatic equilibrium state. Nucl. Eng. Des., 301, 341-352. https://doi.org/10.1016/j.nucengdes.2016.02.025.

4. Stanisz, P., Cetnar, J., \& Domańska, G. (2015). Modeling minor actinide multiple recycling in a lead-cooled fast reactor to demonstrate a fuel cycle without longlived nuclear waste. Nukleonika, 60(3), 581-590. DOI: $10.1515 /$ nuka-2015-0111.

5. Cetnar, J., Stanisz, P., \& Domańska, G. (2013). Adiabatic fuel cycle assessment of LFR core with MOX using MCB system. Study for the LEADER project of European Union's 7th FP EURATOM. Kraków: AGH University, WEiP. (KEJ/2013/4).

6. Cetnar, J., Stanisz, P., \& Domańska, G. (2013). Transition to the adiabatic-LFR: preliminary definition of the start-up core and MA-burning capabilities evaluation. Report for the LEADER project of European Union's 7th FP EURATOM. Kraków: AGH University, WeiP. (KEJ/2013/5).

7. Cetnar, J. (2006). General solution of Bateman equations for nuclear transmutations. Ann. Nucl. Energy, 33, 640-645. DOI: 10.1016/j.anucene.2006.02.004.

8. Cinotti, L., Smith, C. F., Siennicki, J. J., Abderrahim, H. A., Benamati, G., Locatelli, G., Monti, S., Wider, H., Struwe, D., Orden, A., \& Hwang, S. (2007). The potential of the LFR and the ELSY project. In International Congress on Advances in Nuclear Power Plants (ICAPP 2007): The nuclear renaissance at work. Proceedings of a meeting held 13-18 May 2007, Nice, France (Vol. 5, pp. 3231-3240). Societe Francaise d'Energie Nucleaire (SFEN).

9. Mills, R. W. (2007). Future requirements of nuclear data for the handling, reprocessing and disposal of spent nuclear fuel. In International Conference on Nuclear Data for Science and Technology. DOI: 10.1051/ndata:07730.

10. Döderlein, C., Tucek, K., Cetnar, J., Grasso, G., \& Stanisz, P. (2012). Definition of the ELFR core and neutronic characterization, Lead-cooled European Advanced Demonstrator Reactor. European Commission Directorate-General JRC. (DEL 005-2011).

11. OECD NEA Data Bank. (2006). Processing of the JEFF-3.1 Cross Section Library into Continuous Energy Monte Carlo Radiation Transport and Criticality Data Library. (NEA/NSC/DOC18).

12. Firestone, R. B., Shirley, V., Baglin, C., Chu, S., \& Zipkin, J. (1996). Table of isotopes 8E. New York: John Wiley \& Sons, Inc.

13. Mills, R. W. (2008). Nuclear data for the handling, reprocessing and disposal of spent nuclear fuel. UK: Nexia Solutions Ltd., Sellafield Works.

14. Bays, S., Piet, S., Pope, M., Youinou, G., Dumontier, A., \& Hawn, D. (2009). Transmutation dynamics. Impacts of multi-recycling on fuel cycle performances. Idaho National Laboratory. (INL/EXT-09 16857).

15. OECD Nuclear Energy Agency. (2015). Review of integral experiments for minor actinide management. (NEA no. 7222). 